\title{
Lithium depletion in solar-like stars: no planet connection
}

\author{
P. Baumann ${ }^{1}$, I. Ramírez ${ }^{1}$, J. Meléndez ${ }^{2}$, M. Asplund ${ }^{1}$, and K. Lind ${ }^{3}$ \\ 1 Max Planck Institute for Astrophysics, Postfach 1317, 85741 Garching, Germany \\ e-mail: [pbaumann, ivan, asplund] ampa-garching .mpg. de \\ 2 Centro de Astrofisica da Universidade do Porto, Rua das Estrelas, 4150-762 Porto, Portugal \\ e-mail: jorge@astro.up.pt \\ 3 European Southern Observatory(ESO), Karl-Schwarzschild-Str. 2, 85748 Garching, Germany \\ e-mail: klind@eso.org
}

Received 2 June 2010 / Accepted 24 June 2010

\begin{abstract}
We have determined precise stellar parameters and lithium abundances in a sample of 117 stars with basic properties very similar to the Sun. This sample selection reduces biasing effects and systematic errors in the analysis. We estimate the ages of our sample stars mainly from isochrone fitting but also from measurements of rotation period and X-ray luminosity and test the connection between lithium abundance, age, and stellar parameters. We find strong evidence for increasing lithium depletion with age. Our sample includes 14 stars that are known to host planets and it does not support recent claims that planet-host stars have experienced more lithium depletion than stars without planets. We find the solar lithium abundance normal for a star of its age, mass, and metallicity. Furthermore, we analyze published data for 82 stars that were reported to support an enhanced lithium depletion in planet hosts. We show that those stars in fact follow an age trend very similar to that found with our sample and that the presence of giant planets is not related to low lithium abundances. Finally, we discuss the systematic biases that led to the incorrect conclusion of an enhanced lithium depletion in planet-host stars.
\end{abstract}

Key words. Sun: abundances - stars: abundances - planetary systems

\section{Introduction}

The lithium abundances of solar-like stars in the solar neighborhood spread over more than two orders of magnitude, which is much larger than the range of abundances seen for other elements (e.g., Reddy et al. 2003). The Sun, in particular, has a very low lithium abundance compared to many nearby solar analogs (e.g., Lambert \& Reddy 2004). Furthermore, the photospheric solar lithium abundance is about 160 times lower than that measured in meteorites $\left(\log \epsilon_{\mathrm{Li}, \odot}=1.05 \pm 0.10 \mathrm{dex}^{1}\right.$, $\log \epsilon_{\mathrm{Li} \text {,met }}=3.26 \pm 0.05 \mathrm{dex}$; both values are from Asplund et al. 2009). This difference between the current solar and protosolar values is not predicted by standard stellar evolution models (e.g., D’Antona \& Mazzitelli 1984).

The wide range of observed lithium abundances in nearby solar-like stars is most likely due to a dependency between $\log \epsilon_{\mathrm{Li}}$ and the star's age and mass (e.g., Montalbán \& Schatzman 2000; Charbonnel \& Talon 2005; Xiong \& Deng 2009; Do Nascimento et al. 2009). Lithium is easily destroyed by proton capture reactions in stellar interiors. Thus, if lithium is transported between the chemically mixed outer convection zone and deeper lying regions with temperatures that are high enough for lithium destruction, the photospheric abundance will decrease with time. Macroscopic transport processes in the radiative zone below the convective envelope contribute to the lowering of the surface lithium abundance throughout the main-sequence stage. This would explain why the photospheric solar abundance is

1 We use the standard notation $\log \epsilon_{\mathrm{X}}=\log \frac{n_{\mathrm{X}}}{n_{\mathrm{H}}}+12$, where $n_{\mathrm{X}}$ and $n_{\mathrm{H}}$ are the the number densities of element $\mathrm{X}$ and hydrogen, respectively. Also, for metallicities we use the common abbreviation $[\mathrm{Fe} / \mathrm{H}]=$ $\log \epsilon_{\mathrm{Fe}}-\log \epsilon_{\mathrm{Fe}}^{\odot}$. much smaller than the meteoritic one. We expect an enhanced lithium depletion in stars with larger convection zones on the main sequence as well as in stars with a higher degree of differential rotation between the radiative core and the convective envelope (see below). The reason is that lithium is only depleted as it moves to deeper and therefore hotter regions of a star, where the temperature is high enough (about 2.5 million $\mathrm{K}$ ) for proton capture (see, e.g., Pinsonneault 1997).

Recently, it has been suggested that the presence of planets around a star could affect the evolution of the photospheric lithium abundance (e.g., Bouvier 2008). A long-lasting star-disk interaction during the star's pre-main sequence phase could slow down the host-star's rotation and therefore increase the degree of differential rotation between the star's core and envelope. Rotationally-driven mixing is then enhanced, thus destroying more lithium than in stars without planets because fast rotators evolve with little core-envelope decoupling. Planet migration affects the star's angular momentum, which might also have an impact on $\log \epsilon_{\mathrm{Li}}$. Finally, the ingestion of a planet can increase the surface lithium abundance (e.g., Montalbán \& Rebolo 2002; Israelian et al. 2001).

The possibility of a lithium-planet connection is subject of ongoing discussions. Recent work by Gonzalez (2008), Gonzalez et al. (2010), Castro et al. (2008), and Israelian et al. (2009) suggests a possible $\log \epsilon_{\mathrm{Li}}$-planet dependency, whereas Ryan (2000) and Luck \& Heiter (2006) find that stars with planets show the same lithium distribution as the comparison field stars. Takeda et al. (2007, 2010) describe the stellar angular momentum as the crucial factor that determines the lithium abundance of solar-like stars and find that slow rotators show an enhanced lithium depletion. Planets could be the reason for a slow 
rotation, but they were not able to draw firm conclusions due to the low number of planet hosts in their sample and the fact that their use of the star's projected rotational velocity, $v \sin i$, instead of measured rotation periods introduces additional uncertainty, since the inclination angle $i$ is unknown.

From a practical point of view, an enhanced lithium depletion in planet-hosts would be greatly beneficial for the search for extrasolar planets, because all known methods for extrasolar planet detection (e.g., radial velocity, transits, or microlensing) are very time consuming. With an enhanced lithium depletion, however, one could preselect planet-host candidates just by obtaining the stars' chemical composition.

In this paper, we derive lithium abundances and ages for a sample of solar-type stars to examine whether there is a correlation between lithium and age as well as a possible connection between lithium and planets. We also examine lithium abundances and ages for the solar analog sample of Israelian et al. (2009), who claim to have found evidence for an enhanced lithium depletion in planet-host stars.

\section{Observations and analysis}

Our sample consists of 117 solar-like stars selected from the Hipparcos catalog (Perryman et al. 1997) as in Meléndez \& Ramírez (2007). They where observed at the McDonald (Robert G. Tull coudé spectrograph on the $2.7 \mathrm{~m}$ Harlan Smith telescope; RGT), Las Campanas (MIKE spectrograph on the $6.5 \mathrm{~m}$ Magellan Clay telescope), and La Silla (HARPS spectrograph on the $3.6 \mathrm{~m}$ ESO telescope) observatories. Our few solar twins observed at Keck (Meléndez et al. 2006) are not discussed here since they are already included in the McDonald sample.

The RGT and MIKE data (spectra as well as stellar parameters) are from Ramírez et al. (2009, hereafter R09) and Meléndez et al. (2009, 2010, hereafter M09), respectively; two stars (HIP 10215 and HIP 79672) are part of both samples. HARPS spectra for 12 more stars were obtained from the ESO archive, while spectra for 6 other stars were obtained from the $\mathrm{S}^{4} \mathrm{~N}$ database (Allende Prieto et al. 2004) ${ }^{2}$. One of the objects (HIP 80337) occurs in both the HARPS and the $\mathrm{S}^{4} \mathrm{~N}$ samples (we use the HARPS parameters, because they have the smaller uncertainties), so that we have 17 additional stars. The spectra for these stars were analyzed in an identical fashion as in R09 (see below). All sub-samples contain one or more solar reference objects for normalization: R09 used the light reflected from the asteroids Ceres and Vesta, M09 used Vesta, and for the stars added in this work, spectra of asteroid Ceres, Jupiter's moon Ganymedes, and the Moon were used. Table 1 shows the specifications of each sub-sample, where the last two lines describe the additional data from this work. All spectra have a signal-to-noise ratio $(\mathrm{S} / \mathrm{N})$ greater than about 200 , which allows the determination of lithium abundances as low as solar. Note that three stars in our sample are also included in M09 (HIP 79672) and R09 (HIP 14614 and HIP 42438). For the further analysis, we use the parameters with the smaller uncertainties.

The HARPS and $\mathrm{S}^{4} \mathrm{~N}$ data were analyzed by first measuring $\mathrm{Fe} I$ and Fe II equivalent widths (EWs), where a line list of 45 iron lines ( $34 \mathrm{Fe} \mathrm{I}$ and 11 Fe II lines) within the wavelength range from 4445 to $8294 \AA$ was used. The Fe lines have a broad range of excitation potentials (from $\sim 0.1$ to $\sim 4.6 \mathrm{eV}$ ). The line list adopted is from Scott et al. (in prep.; see also

\footnotetext{
2 The Spectroscopic Survey of Stars in the Solar Neighbourhood $\left(\mathrm{S}^{4} \mathrm{~N}\right)$ data and more detailed information can be found at http://hebe.as . utexas. edu/s4n/
}

Table 1. Specifications for the different sub-samples.

\begin{tabular}{ccccc}
\hline \hline $\begin{array}{c}\text { Sample } \\
\text { name }\end{array}$ & $\begin{array}{c}\text { Instrument/ } \\
\text { telescope }\end{array}$ & $\begin{array}{c}\text { Wavelength } \\
\text { coverage } \\
\text { (in } \AA \text { ) }\end{array}$ & $\begin{array}{c}\text { Spectral } \\
\text { resolution } \\
R=\Delta \lambda / \lambda\end{array}$ & $\begin{array}{c}\text { Number } \\
\text { of } \\
\text { stars }\end{array}$ \\
\hline R09 & RGT/McDonald & $3800-9125$ & 60000 & 63 \\
M09 & MIKE/Magellan & $3400-10000$ & 65000 & 42 \\
this & RGT/McDonald, & $3800-9125$ & $45000-$ & 18 \\
work & HARPS/ESO & $3783-6865$ & 110000 & \\
\hline
\end{tabular}

Asplund et al. 2009). To calculate the iron abundances ([Fe/H]) from the $\mathrm{Fe} / \mathrm{Fe}$ II lines, we used the spectrum synthesis program MOOG (Sneden 1973) and ATLAS 9 model atmospheres without convective overshoot (e.g., Kurucz 1993). We iteratively assigned the stellar parameters effective temperature, surface gravity, and microturbulent velocity by forcing simultaneously Fe I excitation equilibrium and Fe I/Fe II ionization equilibrium. For a more detailed description see Ramírez et al. (2009). With the method described above, we derived the following mean errors: $\sigma\left(T_{\text {eff }}\right)=40 \mathrm{~K}, \sigma(\log g)=0.06 \mathrm{dex}$, and $\sigma([\mathrm{Fe} / \mathrm{H}])=$ 0.025 dex.

Stellar ages and masses were determined primarily from the location of stars on the theoretical HR-diagram $\left(T_{\text {eff }}\right.$ vs. $\log g$ ) as compared to theoretical predictions based on stellar evolution calculations (isochrones). We produced a grid of $\mathrm{Y}^{2}$ isochrones (e.g., Yi et al. 2001) with steps of 0.01 dex in metallicity around the solar value. For each star, we computed the age probability distribution of all isochrone points included within a 3- $\sigma$ radius from the observed stellar parameters. The adopted mean age and 1- $\sigma$ Gaussian-like upper and lower limits were derived from the age probability distribution (e.g., Lachaume et al. 1999; Reddy et al. 2003). Although isochrone ages of unevolved stars are typically unreliable, the high precision of our stellar parameters allowed us to infer reasonably accurate absolute isochrone ages, even for stars as young as $\sim 3 \mathrm{Gyr}$; relative ages are naturally even better determined. For most stars younger than about 3 Gyr only upper limits could be determined. For these younger stars, we adopted ages based on measurements of rotational periods (Gaidos et al. 2000; Barnes 2007) and X-ray luminosity (Agüeros et al. 2009) along with the rotation-age relation by Barnes (2007) and the X-ray luminosity-age relation by Guinan \& Engle (2009). In a few cases of stars in the intermediate age region (2-4 Gyr), an average of isochrone and rotational ages was determined to improve the age estimate. For stars with very unreliable isochrone ages we used the activity-based ages by Mamajek \& Hillenbrand (2008) and Saffe et al. (2005). Our adopted ages and the methods to obtain them are listed in Table 4.

Using our stellar parameters as well as those in R09 and M09, we derived the lithium abundances for all 117 stars with line synthesis using $M O O G$. For this purpose we generated a line list from 6697 to $6717 \AA$, i.e. $10 \AA$ around the lithium doublet at $6707.8 \AA$. The whole wavelength range was synthesized with $M O O G$, where hyperfine splitting and the Li-doublet were taken into account. Knowing the basic stellar parameters, we were able to fit the lithium line including the effects of the projected rotational velocity $v \sin i$ and the microturbulent and macroturbulent velocities. We derived lithium abundances with a mean error of $\sigma=0.05$ dex for stars in which the lithium doublet was detected. Our mean of all solar spectra is $\log \epsilon_{\mathrm{Li}}=1.03 \pm 0.04$ dex.

Initially, we derived Li abundances assuming line formation in LTE (local thermal equilibrium), in 1D, hydrostatic, plane parallel ATLAS 9 model atmospheres. Abundance corrections were 
P. Baumann et al.: Lithium depletion in solar-like stars

Table 2. Age and lithium abundance of solar twins in open clusters of near solar metallicity.

\begin{tabular}{cccccc}
\hline \hline Cluster & Age in Gyr & $\log \epsilon_{\mathrm{Li}}$ & $\sigma\left(\log \epsilon_{\mathrm{Li}}\right)$ & {$[\mathrm{Fe} / \mathrm{H}]$} & Source \\
\hline IC 2602 \& IC 2391 & 0.030 & 2.9 & 0.1 & -0.05 & Randich et al. (2001) \\
Pleiades & 0.07 & 2.8 & 0.1 & -0.03 & Soderblom et al. (1993) \\
Blanco 1 & 0.10 & 2.9 & 0.1 & +0.04 & Ford et al. (2005) \\
M 34 (NGC 1039) & 0.25 & 2.8 & 0.1 & +0.07 & Jones et al. (1997) \\
Coma Berenices & 0.6 & 2.4 & 0.15 & -0.05 & Ford et al. (2001) \\
NGC 762 & 2.0 & 2.1 & 0.1 & +0.01 & Sestito et al. (2004) \\
M 67 & 3.9 & 1.2 & 0.5 & +0.05 & Pasquini et al. (2008) \\
\hline
\end{tabular}

Notes. Data are from the compilation by Sestito \& Randich (2005).

thereafter applied to account for departures from LTE in the formation of the Li resonance line. The non-LTE modeling procedure is the same as described in Lind et al. (2009), but extended to cover also super-solar metallicities. For our sample stars, the abundance corrections range from $-0.03 \mathrm{dex}$ to $+0.08 \mathrm{dex}$, depending on the lithium line strength and exact stellar parameters. In stars for which the equivalent width of the lithium line is below $\sim 100 \mathrm{~m} \AA$, over-ionization of neutral lithium results in positive abundances corrections, increasing with increasing metallicity and decreasing effective temperature. When the line starts to become saturated, increased photon losses pushes the statistical equilibrium in the opposite direction, i.e. into overrecombination, resulting in negative corrections for some stars (see Lind et al. 2009, for more details). The non-LTE corrections are very small in comparison to the full range covered in lithium abundance, and hence the qualitative results of this study are the same for lithium abundances inferred in LTE and non-LTE. Note that the NLTE corrections are computed using MARCS models (Gustafsson et al. 2008). Our NLTE corrected solar lithium abundance is $1.07 \pm 0.04$, in good agreement with the 3D-NLTE estimate by Asplund et al. (2009).

Our adopted stellar parameters and derived LTE and nonLTE lithium abundances are given in Table 4. Figure 1 shows the good agreement between the three observational sub-samples, which reduces errors introduced by inhomogeneous data processing and therefore makes the analysis more reliable. It also is a proof of the consistently good quality of the data.

We have also considered the lithium abundances of solar twins from 8 open clusters as listed in Table 2. Data are from the compilation by Sestito \& Randich (2005) as shown in Table 2 with updated data for M 67 by Pasquini et al. (2008). The age for M 67 is taken from VandenBerg et al. (2007) and Yadav et al. (2008), the lithium abundances for M 67 stars are from Castro et al. (2010). The clusters IC 2602 and IC 2391 are listed as one here, because their parameters are basically the same. We only used open clusters around solar metallicity $(0.0 \pm 0.1 \mathrm{dex})$ and with reliable data. The solar twins that we select in open clusters are stars of one solar mass by definition, i.e. they are main sequence stars with $1 M_{\odot}$ based on their effective temperature. We take into account the increase of the solar effective temperature with increasing age in the selection of stars from open clusters.

\section{Results}

\subsection{Lithium and age}

Using our sample of solar-like stars a clear lithium-age trend is observed (Fig. 2). The dependency is as expected: older stars show more lithium depletion. The Spearman correlation coefficient is $r_{\text {tot }}=-0.61$. This trend becomes clearer when we restrict the sample to solar twins, as in Fig. 3. We define solar twins as

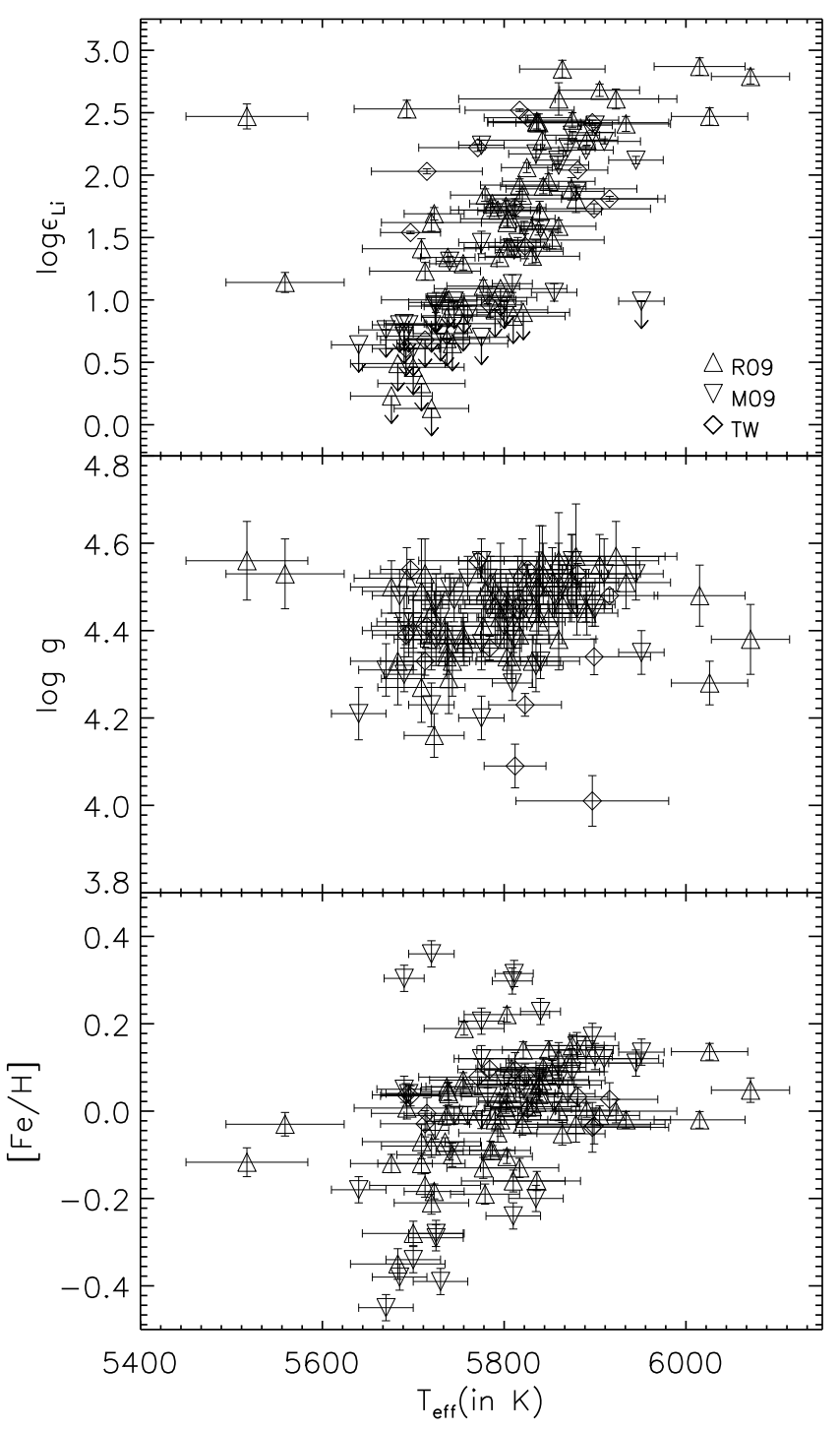

Fig. 1. NLTE lithium abundance, $\log g$, and metallicity plotted against effective temperature. R09 and M09 stand for data from Ramírez et al. (2009) and Meléndez et al. (2009, 2010), respectively; TW is data reanalyzed in this work.

stars with $[\mathrm{Fe} / \mathrm{H}]=0.0 \pm 0.1$ and $M=(1.00 \pm 0.04) M_{\odot}$ The stars from the open clusters given in Table 2 fit the trend in Fig. 3 very well. This is very important, because the ages of these clusters are well determined and the fact that they lie in the midst of the lithium vs. age trend of the field solar twins suggests that the ages we derived for individual stars are reliable. The Spearman correlation coefficient for the solar twin $\log \epsilon_{\mathrm{Li}}$-age trend 


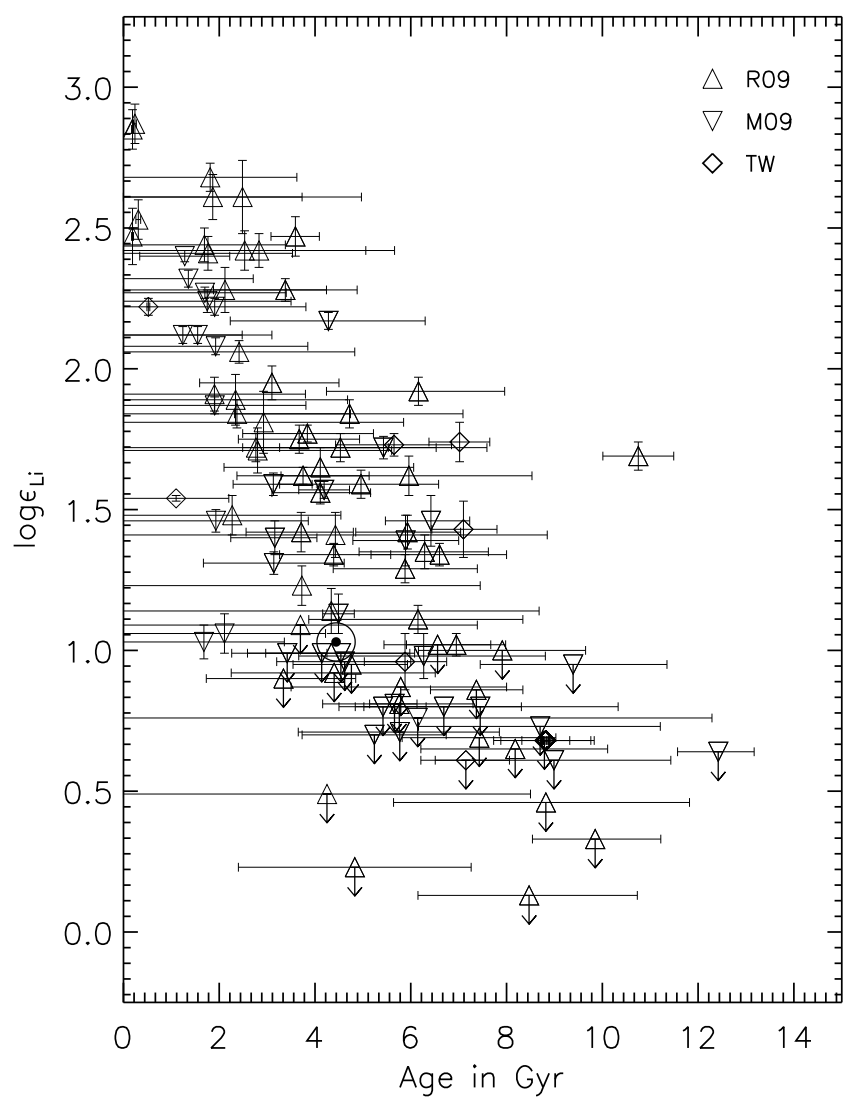

Fig. 2. $\log \epsilon_{\mathrm{Li}}$ vs. age for stars from the three observational sub-samples. Down-arrows denote upper limits. The Sun is marked with $\odot$.

including the open cluster data is $r_{\text {twin }}=-0.75$. Another interesting thing to point out here is the fact that the Sun (marked with $\odot$ in the figures) fits the trend very well. This leads to the conclusion that the Sun does not have a particularly low lithium abundance compared to stars of similar age, mass, and metallicity.

Figure 3 also compares our observational data with model predictions from Charbonnel \& Talon (2005) for different initial rotational velocities of the stars. These hydrodynamical models give predictions for the evolution of surface $\mathrm{Li}$ abundance in solar-type stars, accounting self-consistently for element segregation and transport of angular momentum and chemicals by meridional circulation, shear turbulence and internal gravity waves. They agree not only with the general lithium depletion trend observed by us, but it could also explain the relatively large scatter as a result of differences in initial stellar rotational velocities.

\subsection{Lithium and planets}

In Fig. 4 we plot lithium abundance against age, this time for a sample of metal-rich solar analogs. As metal-rich solar analogs we define stars with $[\mathrm{Fe} / \mathrm{H}]=0.25 \pm 0.15$ and $M=$ $(1.08 \pm 0.08) M_{\odot}$. We use those criteria because our sub-sample of planet-hosts is biased towards those higher metallicities and masses. In this case we make a distinction between stars that are known to host planets (filled symbols) and those for which planets have not yet been detected (open symbols).

We used a two-dimensional Kolmogorov-Smirnov (KS) test to measure the probability that the samples of metal-rich solar analogs with and without planets in Fig. 4 belong to the same

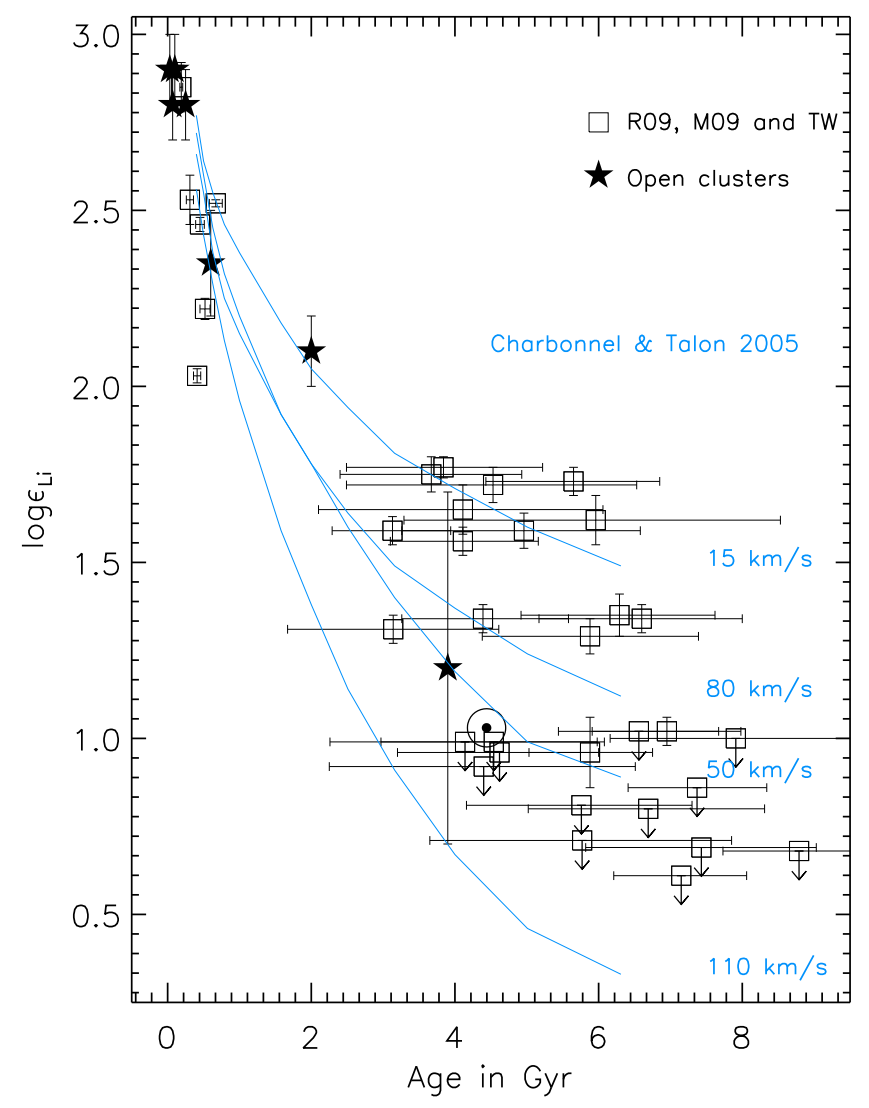

Fig. 3. $\log \epsilon_{\mathrm{Li}}$ vs. age for solar twins from R09, M09, TW and from the solar twins in solar metallicity open clusters. Note the different scale compared to Fig. 2. The solid lines are the predicted values from the models by Charbonnel \& Talon (2005) for different initial rotational velocities.

parent population. Using a Monte Carlo simulation, we took into account the errors in lithium abundance and age by choosing random, normally distributed values within each variable's $1-\sigma$ environment on the linear scale, which means that instead of $\log \epsilon_{\mathrm{Li}}$, we used $10^{\log \epsilon_{\mathrm{Li}}-12}$, that is $\frac{n_{\mathrm{Li}}}{n_{\mathrm{H}}}$. The upper limits were accounted for by distributing the values uniformly between 0 and the upper limit.

We averaged the outcome of $1000 \mathrm{KS}$ tests. This gave a probability for our metal-rich solar analogs with planets and those without planets to be part of the same parent sample of $64 \pm 15 \%$; if we ignore the error bars and upper limits, this probability goes up to $80 \%$. This is very important for the further analysis of the data, because it tells us that there is no intrinsic difference between the two sub-samples. It is highly unlikely that the planet-hosts and comparison stars are different regarding their surface lithium abundance.

The age-lithium correlation coefficient for the solar twins is similar to that corresponding to the metal-rich solar analogs $\left(r_{\text {twin }}=-0.75, r_{\text {analog }}=-0.71\right)$. However, the shapes of those trends are not identical. In the range from 3 to $6 \mathrm{Gyr}$, in particular, it is clear that for a given age, metal-rich solar analogs have on average lower lithium abundances than solar twins (see also Fig. 5c). This is independent of whether the star has a planet or not. The age-lithium trend in Sun-like stars is thus metallicity dependent. This metallicity effect is predicted by stellar models due to the deeper convection zone in more metal-rich stars (Castro et al. 2009) and has lately been confirmed (see, e.g. do Nascimento et al. 2010, Fig. 5). Note, however, that the mass 
P. Baumann et al.: Lithium depletion in solar-like stars

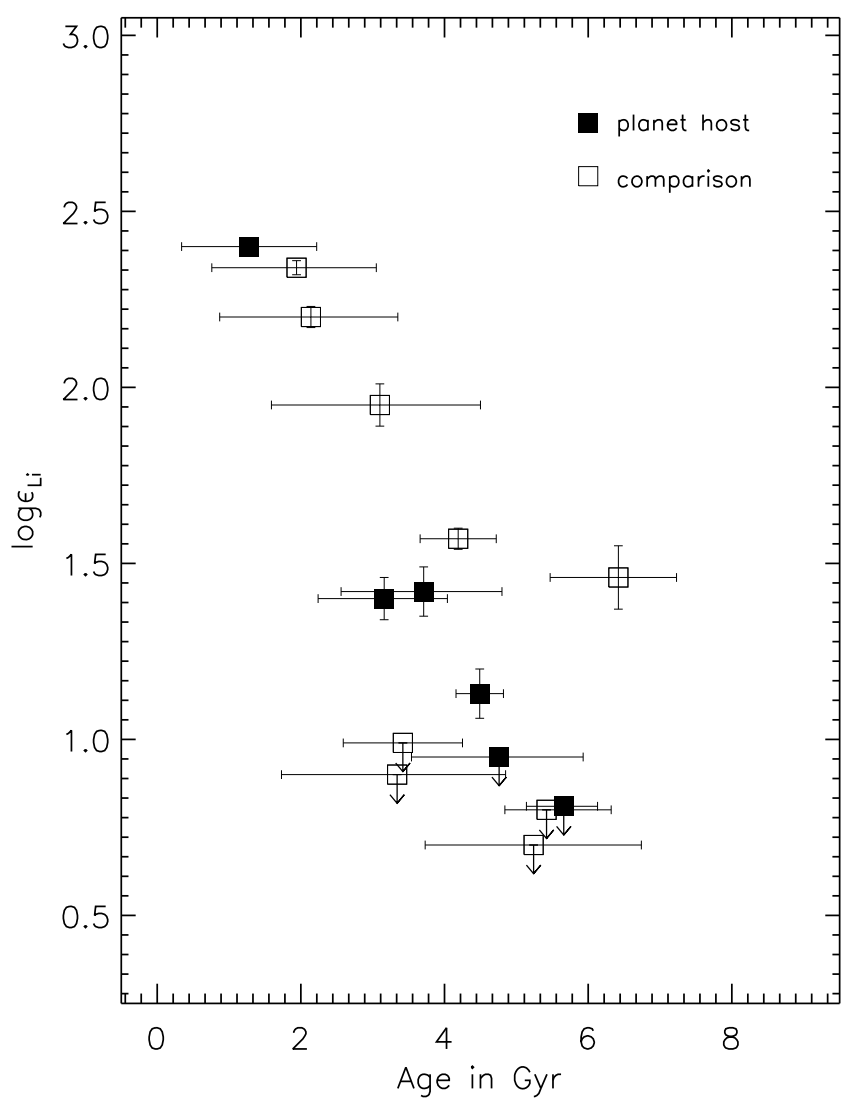

Fig. 4. Same as Fig. 3 but for metal-rich solar analogs $([\mathrm{Fe} / \mathrm{H}]=0.25 \pm$ $0.15, M=1.08 \pm 0.08 M_{\odot}$ ).

ranges being compared are somewhat different, and that this will have an impact on the lithium evolution of those two samples.

\section{Discussion}

Recently, it was claimed that planet-harboring solar-type stars show an enhanced lithium depletion and that lithium surface abundances in Sun-like stars do not correlate with stellar ages (Israelian et al. 2009; Sousa et al. 2010, hereafter I09 and S10, respectively).

In Fig. 5, we plot age versus lithium abundance for all 82 stars used in I09 along with the objects from this work (hereafter $\mathrm{B} 10^{3}$ ). With the stellar parameters adopted by I09 we derived the ages for that sample using the same techniques as for our sample; the ages we derive for the I09 sample are given in Table 5. Figure 5 shows the results for all stars panel (a), the solar twins (panel (b)), and the metal-rich solar analogs (panel (c)). We are using the same selection criteria for solar twins and metal-rich solar analogs as in Sect. 3. The agreement between the age-lithium relation found with our sample and that by I09 is excellent, in particular when looking at the solar twin plot. This shows that the stellar parameters used by I09 (which were derived by Sousa et al. 2008) are essentially on the same scale as ours and therefore the combination of both samples for this analysis does not introduce systematic errors. In fact, for the 10 stars in common between our sample and I09 we find differences of $3 \pm 20 \mathrm{~K}$ in $T_{\text {eff }}, 0.02 \pm 0.04$ in $\log g, 0.003 \pm 0.023$ in $[\mathrm{Fe} / \mathrm{H}]$,

\footnotetext{
${ }^{3}$ For consistency, we used our LTE lithium abundance in this discussion because the I09 work does not take into account nonLTE corrections.
}

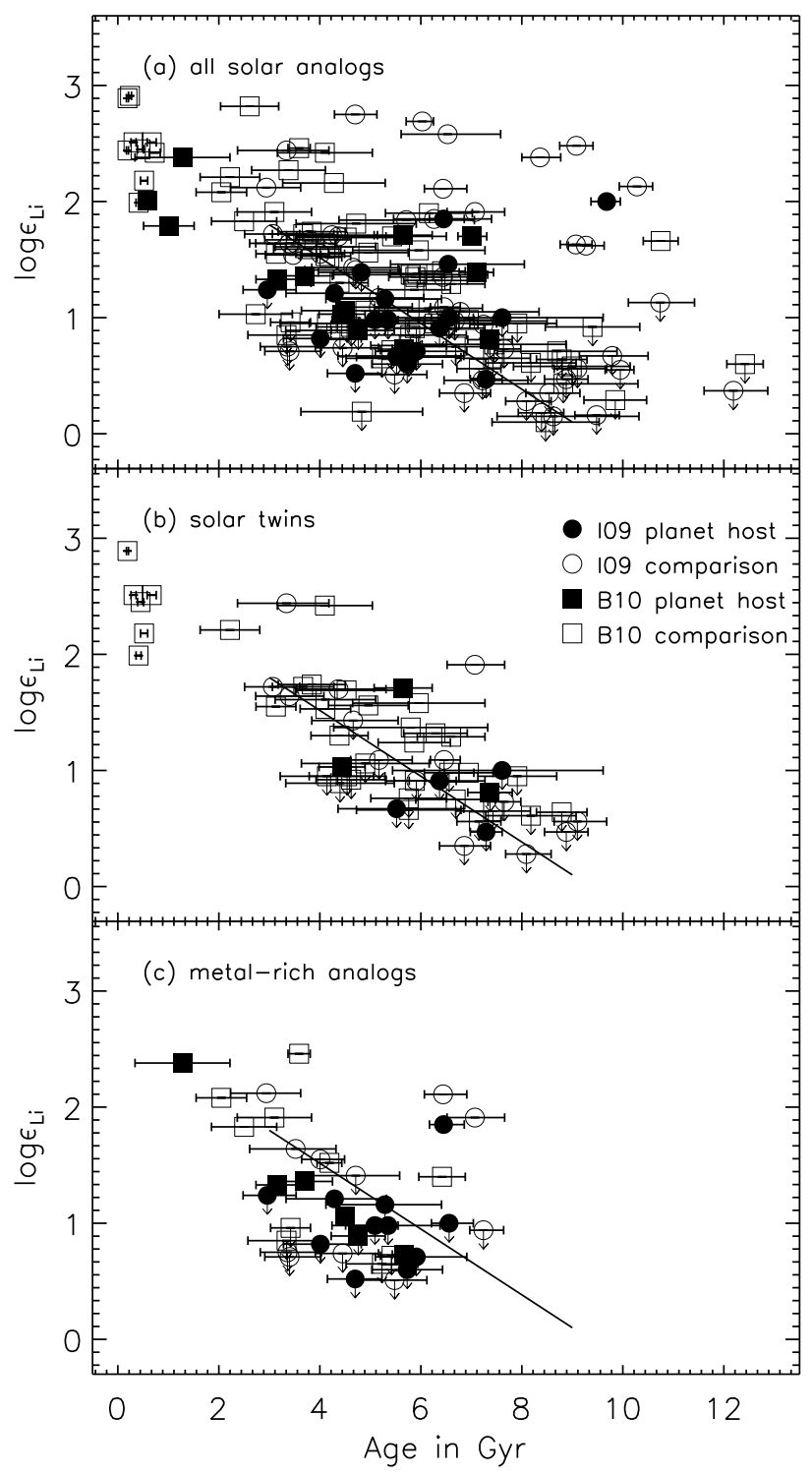

Fig. 5. Comparison between the sample from Israelian et al. (2009, I09) and our sample (B10). The solid line, identically drawn in each panel, is an arbitrary reference line to guide the eye to the different $\log \epsilon_{\mathrm{Li}}$ levels in solar twins an metal-rich solar analogs. Note that for consistency we use the LTE $\log \epsilon_{\mathrm{Li}}$ values here.

and $0.06 \pm 0.11$ in $\log \epsilon_{\mathrm{Li}}$ (the latter for the only 3 stars with lithium doublet detection).

In Fig. 5a, ten stars with ages greater than 4 Gyr and higher than average $\log \epsilon_{\mathrm{Li}}$ can be seen above the main locus. Taking a closer look at those "outliers", the most interesting fact is that they have a particularly low surface gravity $(\log g \simeq 4.1)$ compared to the rest of stars. In Fig. 6, we show $\log \epsilon_{\mathrm{Li}}$ vs. $\log g$. The surface lithium abundance on the $\operatorname{low}-\log g$ side does not follow the main track, for which $\log \epsilon_{\mathrm{Li}}$ decreases with lower surface gravity, which is essentially the age effect, given that all these stars have similar masses. To exclude the possibility of systematic errors in the analysis of the low $\log g$ outliers, we compared the parameters and in particular their ages with various sources (see Table 3). Our derived ages for these outliers are in reasonably good agreement with respect to the the values given in the literature. Only two stars appear to be older than the ages given in the consulted references, but even that difference is only around 2 Gyr and therefore not big enough for these stars to 
Table 3. Ages, masses, and lithium abundances for the outliers in Fig. 5a.

\begin{tabular}{|c|c|c|c|c|}
\hline Object & Age in Gyr & mass in $M_{\odot}$ & $\log \epsilon_{\mathrm{Li}}$ in dex & Ages from other sources in $\mathrm{Gyr}^{a}$ \\
\hline HD 221420 & 4.70 & 1.30 & 2.75 & $\begin{array}{c}.5(\mathrm{GCS}), 4.1(\mathrm{VF} 05) \\
5.1(\mathrm{RP} 98)\end{array}$ \\
\hline HD 114613 & 6.03 & 1.19 & 2.69 & $\begin{array}{l}5.1 \text { (RP98), } 5.6 \text { (B07, rot), } \\
4.9 \text { (VF05), } 4.9 \text { (RP98) }\end{array}$ \\
\hline HD 2151 & 6.53 & 1.12 & 2.58 & 5.2 (GCS), 5.8 (VF05), 6.7 (V05) \\
\hline HD 215456 & 8.36 & 1.04 & 2.38 & $7.3(\mathrm{GCS}), 7.0(\mathrm{I} 02)$ \\
\hline HD 32724 & 9.07 & 0.97 & 1.63 & $9.9(\mathrm{GCS})$ \\
\hline HD 4307 & 9.08 & 1.01 & 2.48 & $\begin{array}{c}7.8\left(\mathrm{~W} 04, R_{H K}^{\prime}, \text { rot }\right), \\
7.4(\mathrm{GCS}), 6.4(\mathrm{VF} 05)\end{array}$ \\
\hline HD 78612 & 9.27 & 0.96 & 1.62 & $8.8(\mathrm{GCS})$ \\
\hline HD 114729 & 9.68 & 0.97 & 2.00 & $\begin{array}{c}10.9 \text { (GCS), } 6.45 \text { (RP98) } \\
\text { [planet-host] }\end{array}$ \\
\hline HD 145809 & 10.28 & 0.96 & 2.13 & $\begin{array}{c}\left.6.9 \text { (W04, } R_{H K}^{\prime}, \text { rot }\right) \\
7.9 \text { (GCS), } 7.4 \text { (VF05) }\end{array}$ \\
\hline HD 32923 & 10.75 & 0.96 & 1.66 & $\begin{array}{l}9.0 \text { (VF05), } 6.2 \text { (W04), } \\
9.9(\mathrm{GCS}),>9.5(\mathrm{~S} 83)\end{array}$ \\
\hline
\end{tabular}

Notes. $R_{H K}^{\prime}$ denotes ages derived from chromospheric activity, rot denotes ages derived from rotation periods. ${ }^{(a)}$ The abbreviations used here are the following: GCS: The Geneva-Copenhagen survey, Nordström et al. (2004), VF05: Valenti \& Fischer (2005), RP98: Rocha-Pinto \& Maciel (1998), V05: Vardavas (2005), I02: Ibukiyama \& Arimoto (2002), W04: Wright et al. (2004) and S83: Soderblom (1983).

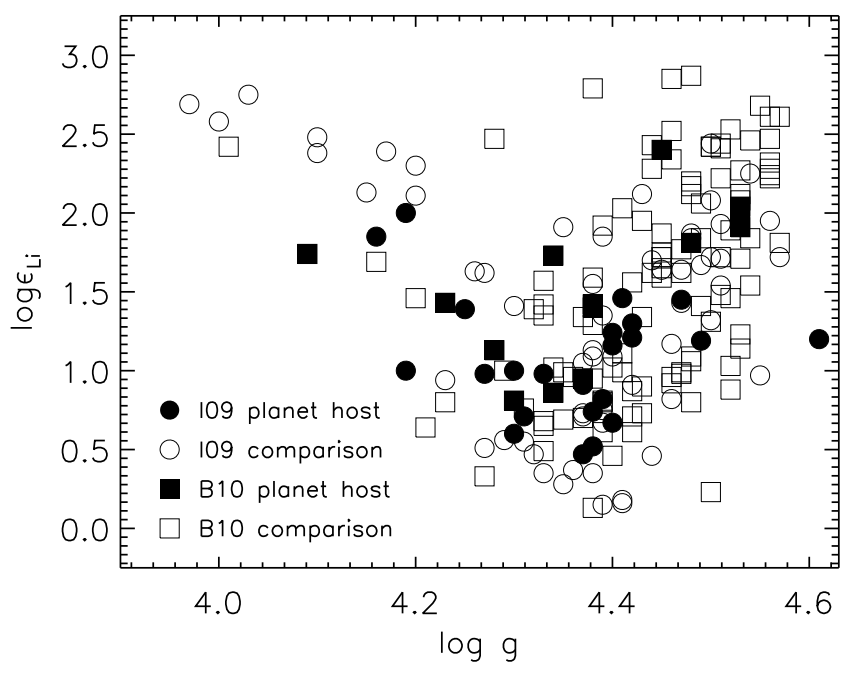

Fig. 6. $\log \epsilon_{\mathrm{Li}}$ vs. $\log g$ for the stars from our sample (B10) and I09.

cease being outliers. This leads us to the conclusion that the ages we derived for the I09 sample and the stellar parameters adopted by I09 (mostly derived by Sousa et al. 2008) are correct and the high-lithium envelope in the lithium-age plane is most likely real. Thus, when restricted to a narrow range of $T_{\text {eff }}$ around the solar value, $\log g \simeq 4.1$ stars have higher lithium abundances than less evolved stars of similar age.

We have examined the results by $\mathrm{S} 10$, who claim that there is no correlation between lithium and age in the I09 sample. The S10 sample is basically the same as in I09, but reduced to the 60 stars studied in Sousa et al. (2008) because of the high homogeneity of the stellar parameters. We show their lithiumage trends in Fig. 7. The selection criteria we used for the solar twins and the metal-rich solar analogs are the same as in Fig. 5. However, this time we are using the masses and ages determined by S10. Although their full sample appears to show no correlation (Fig. 7a), the solar twin sample shows a clear trend between lithium and age. Note that the one planet-host in this sample has a low lithium abundance because of its old

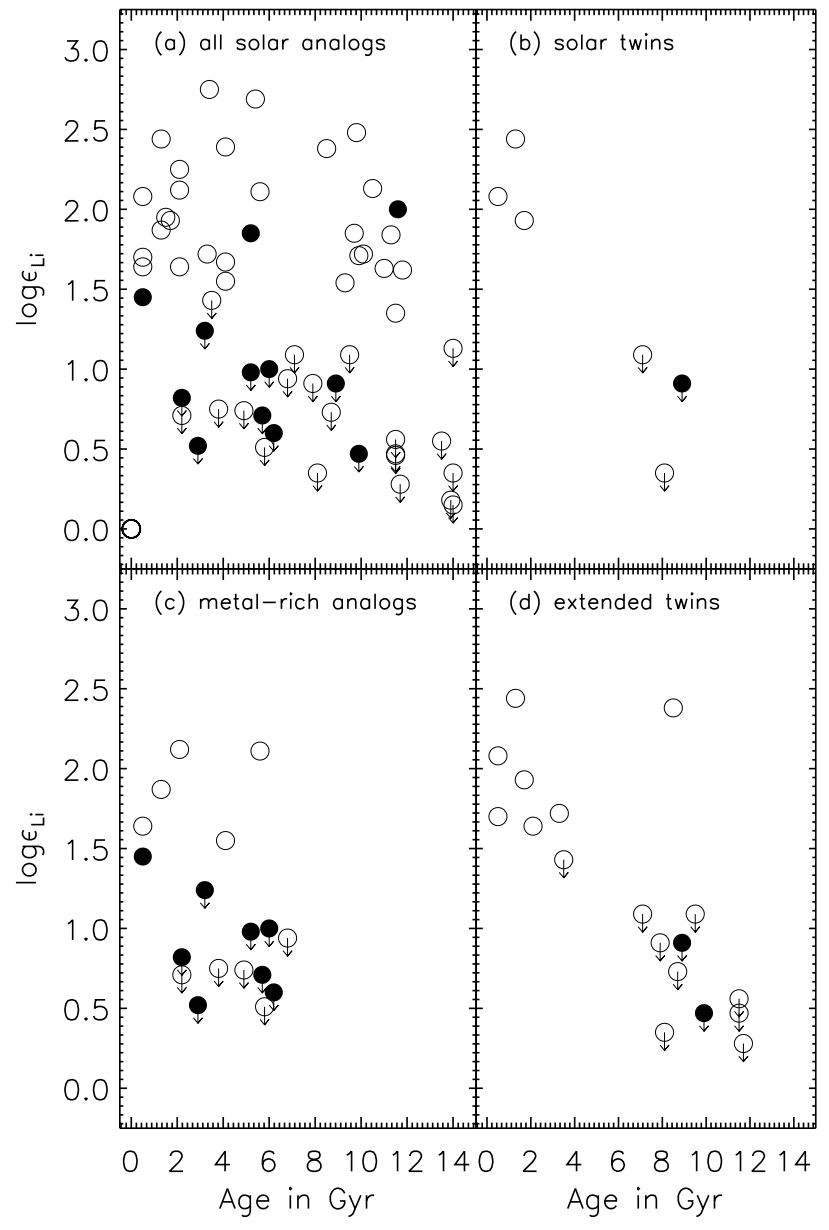

Fig. 7. $\log \epsilon_{\mathrm{Li}}$ vs. age for the $\mathrm{S} 10$ sample. The selection criteria for the four panels are given in the text. Masses and ages adopted to make this figure are from S10.

age and not the fact that it hosts a planet. There is no clear correlation between lithium and age for the metal-rich solar analog 


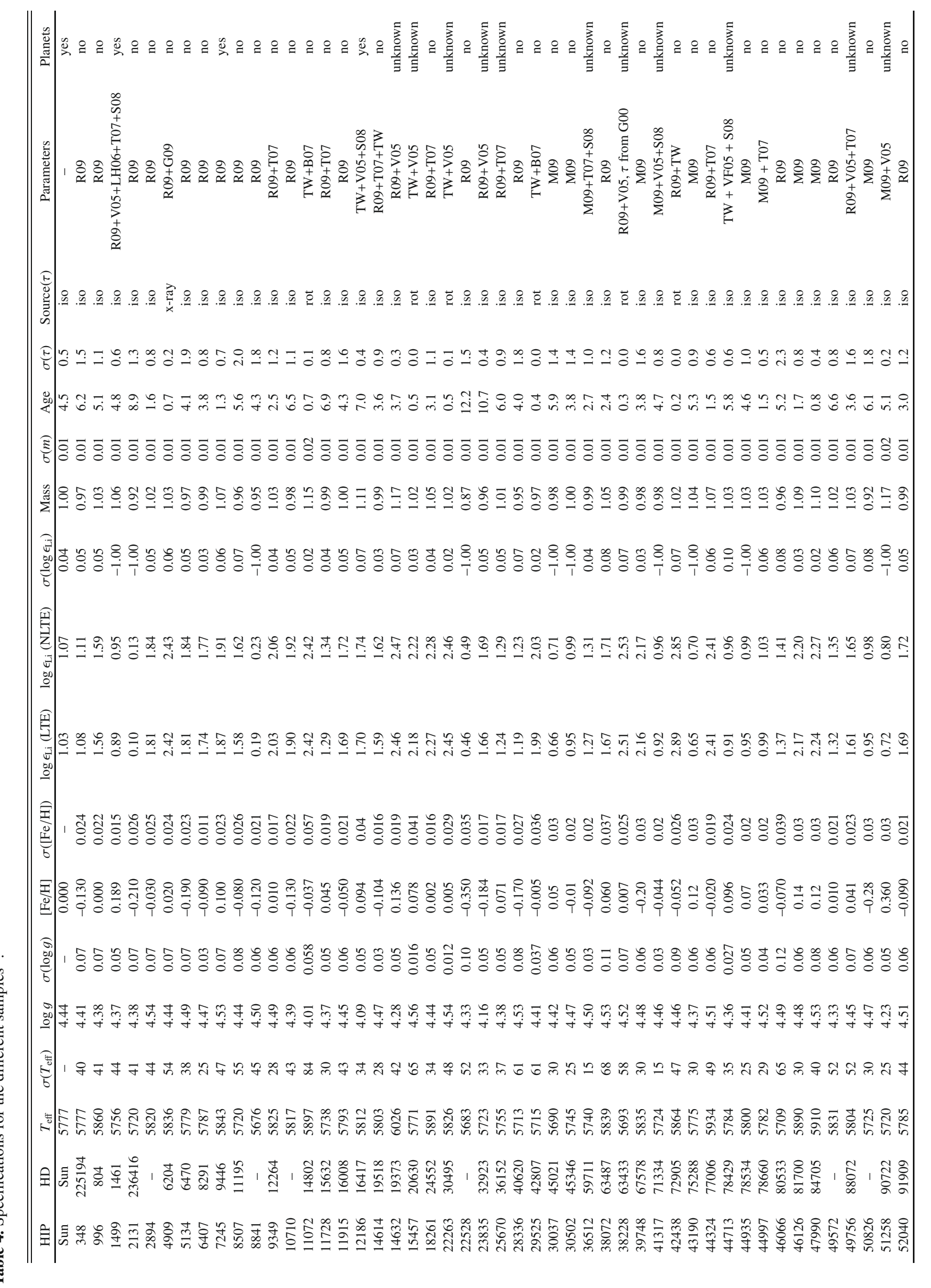




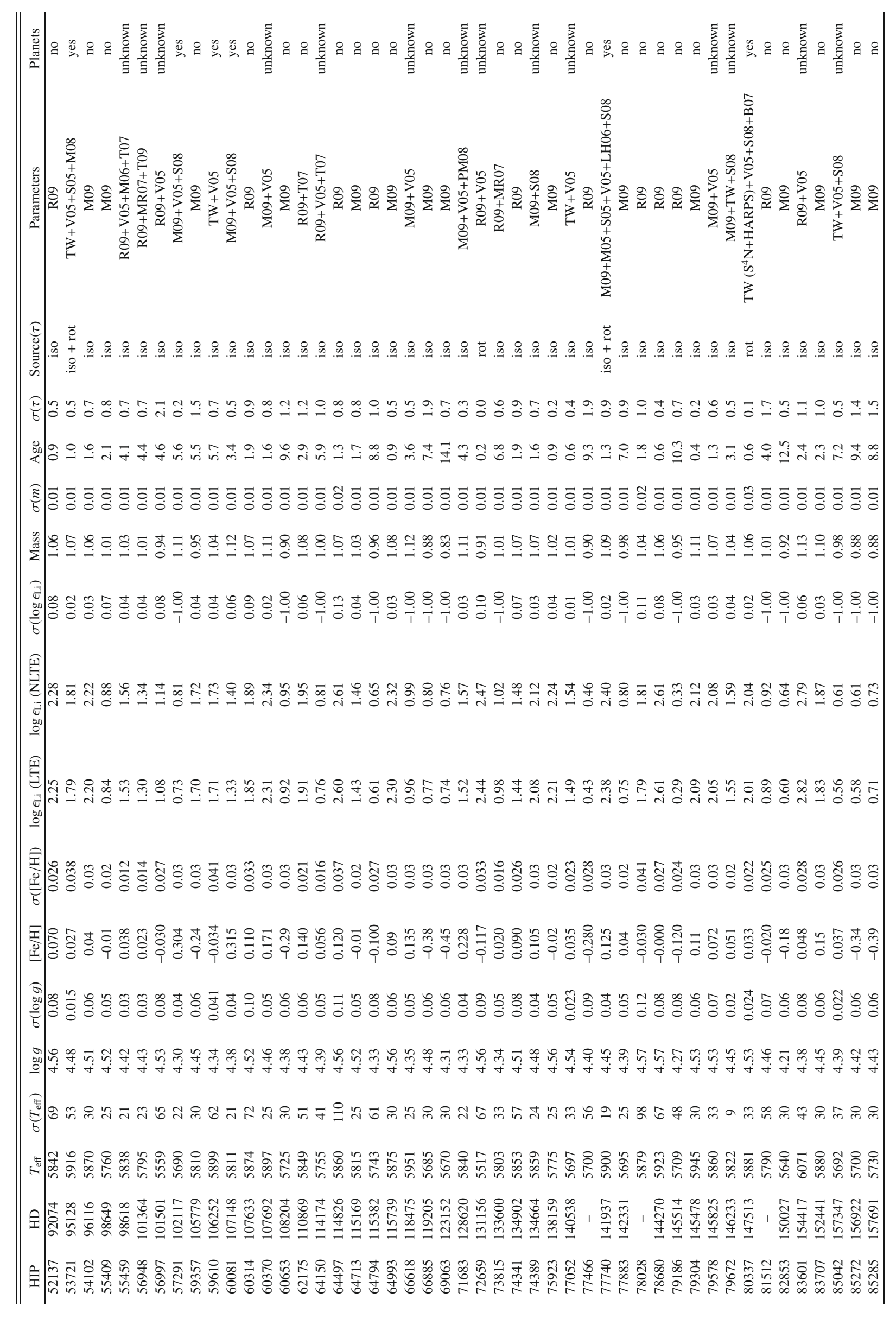




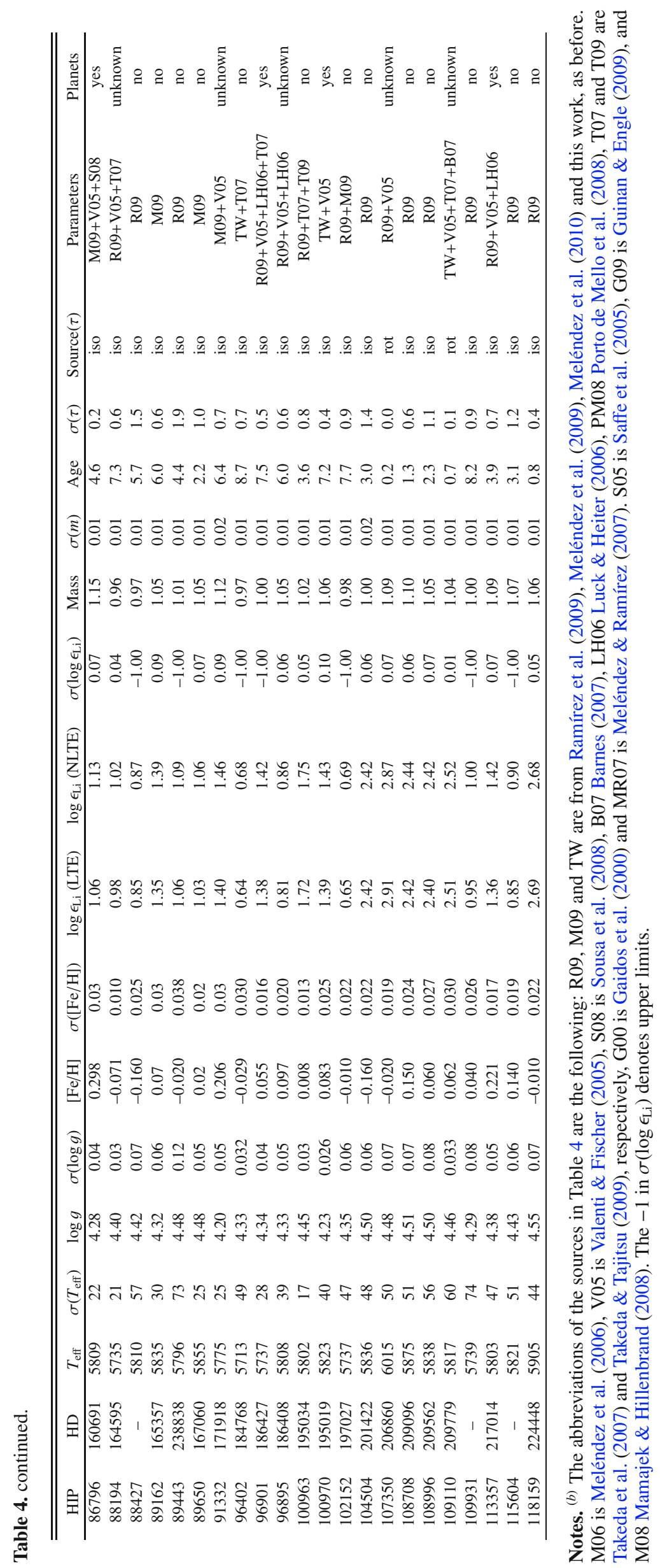


Table 5. Sample used in I09. Masses and ages are from this work.

\begin{tabular}{|c|c|c|c|c|c|c|c|c|c|c|c|}
\hline Star name or HIP & HD & Mass & $\sigma(m)$ & Age & $\sigma(\tau)$ & Star name or HIP & HD & Mass & $\sigma(m)$ & Age & $\sigma(\tau)$ \\
\hline WASP 5 & - & 0.99 & 0.06 & 7.9 & 3.3 & 52409 & 92788 & 1.08 & 0.01 & 3.8 & 1.0 \\
\hline XO-1 & - & 1.01 & 0.01 & 2.3 & 1.2 & 53837 & 95521 & 0.98 & 0.01 & 3.4 & 1.1 \\
\hline 1499 & 1461 & 1.07 & 0.01 & 4.5 & 0.6 & 54287 & 96423 & 1.01 & 0.01 & 7.2 & 0.6 \\
\hline 1954 & 2071 & 0.97 & 0.01 & 4.6 & 1.1 & 54400 & 96700 & 0.97 & 0.01 & 6.6 & 0.6 \\
\hline 2021 & 2151 & 1.12 & 0.08 & 6.7 & 1.4 & 97998 & 97998 & 0.90 & 0.01 & 1.9 & 0.6 \\
\hline 5339 & 4307 & 1.01 & 0.01 & 9.0 & 0.4 & 60081 & 107148 & 1.12 & 0.01 & 3.0 & 0.6 \\
\hline 6455 & 8406 & 0.98 & 0.01 & 3.0 & 0.6 & 60729 & 108309 & 1.05 & 0.01 & 7.5 & 0.3 \\
\hline 8798 & 11505 & 0.93 & 0.01 & 8.6 & 0.6 & 62345 & 111031 & 1.10 & 0.01 & 3.4 & 0.8 \\
\hline 9381 & 12387 & 0.91 & 0.01 & 9.1 & 1.7 & 64408 & 114613 & 1.20 & 0.02 & 5.9 & 0.3 \\
\hline 9683 & 12661 & 1.10 & 0.03 & 4.5 & 1.3 & 64459 & 114729 & 0.97 & 0.01 & 9.7 & 0.2 \\
\hline 12048 & 16141 & 1.09 & 0.01 & 6.9 & 0.3 & 64550 & 114853 & 0.92 & 0.01 & 7.4 & 0.9 \\
\hline 12186 & 16417 & 1.12 & 0.01 & 6.7 & 0.2 & 65036 & 115585 & 1.13 & 0.03 & 5.3 & 0.5 \\
\hline 14501 & 19467 & 0.94 & 0.01 & 10.0 & 0.3 & 71683 & 128620 & 1.17 & 0.07 & 4.1 & 1.5 \\
\hline 15442 & 20619 & 0.94 & 0.01 & 3.9 & 1.2 & 74500 & 134987 & 1.10 & 0.02 & 5.4 & 0.5 \\
\hline 15330 & 20766 & 0.94 & 0.02 & 3.4 & 1.7 & 78330 & 143114 & 0.88 & 0.01 & 9.9 & 0.8 \\
\hline 15527 & 20782 & 0.98 & 0.01 & 7.3 & 0.3 & 78459 & 143761 & 0.98 & 0.02 & 6.1 & 2.6 \\
\hline 16365 & 21938 & 0.86 & 0.01 & 10.8 & 0.7 & 79524 & 145809 & 0.96 & 0.01 & 10.3 & 0.3 \\
\hline 19925 & 27063 & 1.01 & 0.01 & 4.2 & 1.2 & 79672 & 146233 & 1.03 & 0.01 & 3.3 & 0.8 \\
\hline 20625 & 28471 & 0.97 & 0.01 & 7.7 & 0.3 & 83906 & 154962 & 1.22 & 0.03 & 4.7 & 0.8 \\
\hline 20677 & 28701 & 0.89 & 0.01 & 9.5 & 0.5 & 160691 & 160691 & 1.14 & 0.02 & 4.8 & 0.3 \\
\hline 23627 & 32724 & 0.97 & 0.01 & 9.2 & 0.3 & 95962 & 183658 & 1.01 & 0.01 & 5.3 & 0.7 \\
\hline 22504 & 34449 & 1.02 & 0.01 & 1.5 & 0.8 & 96901 & 186427 & 1.02 & 0.02 & 5.0 & 1.9 \\
\hline 25670 & 36152 & 1.05 & 0.01 & 2.6 & 0.9 & 97336 & 187123 & 1.07 & 0.01 & 3.5 & 1.5 \\
\hline 26737 & 37962 & 0.94 & 0.01 & 5.2 & 1.8 & 97769 & 188015 & 1.10 & 0.02 & 1.8 & 0.9 \\
\hline 27435 & 38858 & 0.95 & 0.01 & 3.3 & 0.7 & 98959 & 189567 & 0.92 & 0.01 & 8.4 & 0.4 \\
\hline 30243 & 44420 & 1.11 & 0.01 & 3.5 & 0.6 & 98589 & 189625 & 1.09 & 0.01 & 2.5 & 1.0 \\
\hline 30104 & 44594 & 1.08 & 0.00 & 4.1 & 0.5 & 102664 & 198075 & 0.99 & 0.01 & 2.3 & 1.0 \\
\hline 30476 & 45289 & 0.97 & 0.00 & 8.8 & 0.3 & 104903 & 202206 & 1.09 & 0.01 & 1.4 & 0.6 \\
\hline 34065 & 53705 & 0.97 & 0.01 & 6.8 & 2.3 & 106006 & 204313 & 1.06 & 0.01 & 4.6 & 0.5 \\
\hline 36512 & 59711A & 0.96 & 0.01 & 5.3 & 1.0 & 108468 & 208704 & 0.99 & 0.01 & 6.6 & 0.3 \\
\hline 39417 & 66428 & 1.09 & 0.02 & 5.8 & 1.0 & 109821 & 210918 & 0.96 & 0.01 & 8.2 & 0.4 \\
\hline 43726 & 76151 & 1.05 & 0.01 & 1.5 & 0.5 & 110109 & 211415 & 0.96 & 0.01 & 6.5 & 1.2 \\
\hline 43686 & 76700 & 1.17 & 0.07 & 4.5 & 1.2 & 112414 & 215456 & 1.04 & 0.01 & 8.4 & 0.4 \\
\hline 44713 & 78429 & 1.02 & 0.01 & 7.0 & 0.5 & 113357 & 217014 & 1.08 & 0.02 & 3.4 & 1.6 \\
\hline 44890 & 78538 & 1.01 & 0.01 & 2.5 & 1.1 & - & 219542 & 1.04 & 0.02 & 4.6 & 1.5 \\
\hline 44860 & 78558 & 0.85 & 0.01 & 12.5 & 0.7 & 115577 & 220507 & 0.98 & 0.01 & 9.3 & 0.5 \\
\hline 44896 & 78612 & 0.96 & 0.01 & 9.4 & 0.3 & 116250 & 221420 & 1.29 & 0.06 & 4.7 & 0.7 \\
\hline 46007 & 81110 & 1.11 & 0.01 & 0.4 & 0.1 & 116852 & 222480 & 1.15 & 0.03 & 5.6 & 0.8 \\
\hline 49728 & 88084 & 0.97 & 0.01 & 6.2 & 0.8 & 116906 & 222582 & 0.99 & 0.01 & 6.7 & 0.8 \\
\hline 50534 & 89454 & 1.03 & 0.01 & 3.0 & 1.1 & 117320 & 223171 & 1.09 & 0.01 & 6.7 & 0.3 \\
\hline 52369 & 92719 & 1.01 & 0.01 & 1.6 & 0.9 & 118123 & 224393 & 0.92 & 0.01 & 3.6 & 1.0 \\
\hline
\end{tabular}

sample in this case, but this could be due to uncertain ages. Since the solar twin sample includes only 6 stars, we define another sample of "extended solar twins" with $[\mathrm{Fe} / \mathrm{H}]=0.0 \pm 0.1$ and $M=1.00 \pm 0.10 M_{\odot}$. The resulting figure shows a very definite trend of $\log \epsilon_{\mathrm{Li}}$ with age and only a single outlier appears. This outlier (HD 215456), however, shows a relatively low $\log g$ of 4.10 (and an almost solar mass of $1.04 M_{\odot}$ ).

We have also examined the lithium vs. effective temperature diagram presented by I09. As shown in Fig. 8a, they found that almost all stars with a high lithium abundance $\left(\log \epsilon_{\mathrm{Li}} \gtrsim\right.$ 1.5 dex) have not been shown to be planet hosts yet, whereas planet hosts and objects where no planets have been found are distributed quite equally below that lithium abundance, although the high number of upper limits makes a direct comparison difficult. In order to make a more robust comparison, we have restricted the comparison sample using the following criteria: we only considered comparison objects within a $2 \sigma$ range in $[\mathrm{Fe} / \mathrm{H}]$, $\log g$, and $T_{\text {eff }}$ around planet hosts, where $\sigma$ are the average values of the uncertainties in the stellar parameters given by Sousa et al. (2008). In this way, we make sure that all stars lie within the same region of parameter space and are not influenced by the age or metallicity effects we find. Note that we do not restrict the lithium range, only metallicity, surface gravity and effective temperature. Using this selection allows for a homogeneous and unbiased comparison. When we restrict the parameter range covered by the comparison stars as described above, the lithium-planet connection disappears; as seen in Fig. 8b, it is not possible to conclude on stronger lithium depletion in planet hosts compared to other stars. We stress that this figure is plotted directly from the I09 data without further manipulation or use of new parameters.

Three systematic biases have led I09 and S10 to conclude that solar-type planet-hosts feature an enhanced lithium depletion and that there is no age dependence:

1. at $[\mathrm{Fe} / \mathrm{H}] \simeq 0.0$, the existing HARPS sample of solar analogs with planets are on average older and therefore more depleted in lithium than non-planet-hosts, but not because they have planets;

2. at higher $[\mathrm{Fe} / \mathrm{H}]$, where most of the $\mathrm{I09}$ planet-hosts concentrate, there is a slightly different $\log \epsilon_{\mathrm{Li}}$ vs. age trend such that, at a given age in the 3-6 Gyr range, metal-rich solar analogs are more lithium-poor compared to solar metallicity ones. This is true for both planet-hosts as well as single stars; 


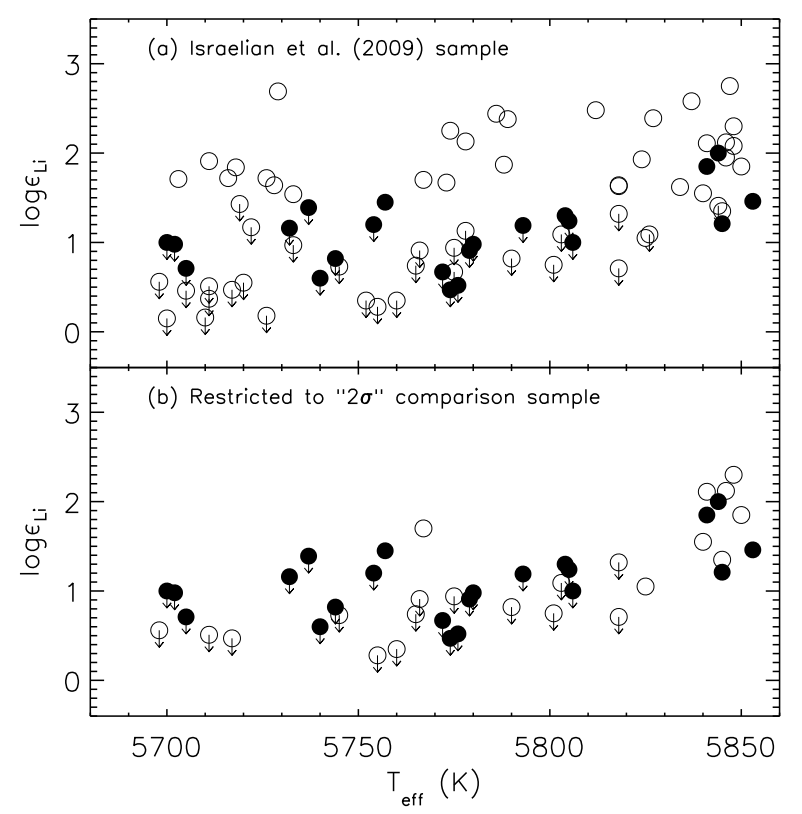

Fig. 8. Lithium abundance as a function of $T_{\text {eff }}$ in stars with and without detected planets from the I09 sample. Filled and open circles represent stars with and without detected planets, respectively. In the upper panel the original comparison made is shown, which is not appropriate because the sample being compared span different ranges in evolutionary phases and metallicities. A less biased comparison is shown in the bottom panel, where we only plot stars without detected planets with stellar parameters $\left(T_{\text {eff }}, \log g,[\mathrm{Fe} / \mathrm{H}]\right)$ within $2 \sigma$ of the planet-hosting stars. When a proper comparison is made, i.e., using stars with similar fundamental parameters, lithium is not abnormally low in stars with detected giant planets.

3. I09 and S10 samples include a number of peculiarly high lithium abundance and relatively $\operatorname{low} \log g(\simeq 4.1)$ stars; only one of them is a planet host.

The apparently lower lithium abundances of planet-hosts found by I09 can thus be fully explained by a combination of age and metallicity effects, not separately but through the age vs. lithium relation.

\section{Conclusions}

1. In stars of solar mass and solar metallicity, it is clear that older stars have experienced more surface lithium depletion. Both the overall lithium-age trend as well as the scatter that we observe in this sample of stars can be explained by the theoretical models by Charbonnel \& Talon (2005).

2. Metal-rich $([\mathrm{Fe} / \mathrm{H}] \sim 0.25)$ solar analogs $\left(M \sim 1.08 M_{\odot}\right)$ also exhibit a lithium-age trend, which is different from that seen in $1 M_{\odot},[\mathrm{Fe} / \mathrm{H}]=0.0$ stars. At any given age in the 3 to 6 Gyr range, the metal-rich solar analogs are more lithiumpoor. This is true for both planet-hosts and single stars.

3. For solar-like stars, the lithium vs age trends for planet-hosts and stars where no planets have been found are statistically identical. Thus, the presence of a planet does not influence the observed surface lithium abundance.

4. A number of solar-like stars with unusually high lithium abundance for their age are present in the field. We note that all of them have relatively low $\log g \simeq 4.1$. We intend to pursue further observational work to better understand this small group of relatively low surface gravity and peculiarly high lithium abundance.

Acknowledgements. We thank G. Israelian for sending us the lithium abundance data from I09.

\section{References}

Agüeros, M. A., Anderson, S. F., Covey, K. R., et al. 2009, ApJS, 181, 444 Allende Prieto, C., Barklem, P. S., Lambert, D. L., \& Cunha, K. 2004, A\&A, 420,183

Asplund, M., Grevesse, N., Sauval, A. J., \& Scott, P. 2009, ARA\&A, 47, 481

Barnes, S. A. 2007, ApJ, 669, 1167

Bouvier, J. 2008, A\&A, 489, L53

Castro, M., Vauclair, S., Richard, O., \& Santos, N. C. 2008, Mem. Soc. Astron. Ital., 79, 679

Castro, M., Vauclair, S., Richard, O., \& Santos, N. C. 2009, A\&A, 494, 663

Castro, M., do Nascimento, J. D., Biazzo, K., Melendez, J., \& de Medeiros, J. R. 2010, A\&A, submitted

Charbonnel, C., \& Talon, S. 2005, Science, 309, 2189

D’Antona, F., \& Mazzitelli, I. 1984, A\&A, 138, 431

Do Nascimento, Jr., J. D., Castro, M., Meléndez, J., et al. 2009, A\&A, 501, 687

do Nascimento, Jr, J. D., da Costa, J. S., \& De Medeiros, J. R. 2010 [arXiv: 1006.3861$]$

Ford, A., Jeffries, R. D., James, D. J., \& Barnes, J. R. 2001, A\&A, 369, 871

Ford, A., Jeffries, R. D., \& Smalley, B. 2005, MNRAS, 364, 272

Gaidos, E. J., Henry, G. W., \& Henry, S. M. 2000, AJ, 120, 1006

Gonzalez, G. 2008, MNRAS, 386, 928

Gonzalez, G., Carlson, M. K., \& Tobin, R. W. 2010, MNRAS, 403, 1368

Guinan, E. F., \& Engle, S. G. 2009, in IAU Symp., 258, 395

Gustafsson, B., Edvardsson, B., Eriksson, K., et al. 2008, A\&A, 486, 951

Ibukiyama, A., \& Arimoto, N. 2002, A\&A, 394, 927

Israelian, G., Santos, N. C., Mayor, M., \& Rebolo, R. 2001, Nature, 411, 163

Israelian, G., Delgado Mena, E., Santos, N. C., et al. 2009, Nature, 462, 189

Jones, B. F., Fischer, D., Shetrone, M., \& Soderblom, D. R. 1997, AJ, 114, 352

Kurucz, R. L. 1993, VizieR Online Data Catalog, 6039, 0

Lachaume, R., Dominik, C., Lanz, T., \& Habing, H. J. 1999, A\&A, 348, 897

Lambert, D. L., \& Reddy, B. E. 2004, MNRAS, 349, 757

Lind, K., Asplund, M., \& Barklem, P. S. 2009, A\&A, 503, 541

Luck, R. E., \& Heiter, U. 2006, AJ, 131, 3069

Mamajek, E. E., \& Hillenbrand, L. A. 2008, ApJ, 687, 1264

Meléndez, J., \& Ramírez, I. 2007, ApJ, 669, L89

Meléndez, J., Dodds-Eden, K., \& Robles, J. A. 2006, ApJ, 641, L133

Meléndez, J., Asplund, M., Gustafsson, B., \& Yong, D. 2009, ApJ, 704, L66

Meléndez, J., Ramírez, I., Casagrande, L., et al. 2010, Ap\&SS, 328, 193

Montalbán, J., \& Schatzman, E. 2000, A\&A, 354, 943

Montalbán, J., \& Rebolo, R. 2002, A\&A, 386, 1039

Nordström, B., Mayor, M., Andersen, J., et al. 2004, A\&A, 418, 989

Pasquini, L., Biazzo, K., Bonifacio, P., Randich, S., \& Bedin, L. R. 2008, A\&A, 489, 677

Perryman, M. A. C., Lindegren, L., Kovalevsky, J., et al. 1997, A\&A, 323, L49 Pinsonneault, M. 1997, ARA\&A, 35, 557

Porto de Mello, G. F., Lyra, W., \& Keller, G. R. 2008, A\&A, 488, 653

Ramírez, I., Meléndez, J., \& Asplund, M. 2009, A\&A, 508, L17

Randich, S., Pallavicini, R., Meola, G., Stauffer, J. R., \& Balachandran, S. C. 2001, A\&A, 372, 862

Reddy, B. E., Tomkin, J., Lambert, D. L., \& Allende Prieto, C. 2003, MNRAS, 340, 304

Rocha-Pinto, H. J., \& Maciel, W. J. 1998, MNRAS, 298, 332

Ryan, S. G. 2000, MNRAS, 316, L35

Saffe, C., Gómez, M., \& Chavero, C. 2005, A\&A, 443, 609

Sestito, P., \& Randich, S. 2005, A\&A, 442, 615

Sestito, P., Randich, S., \& Pallavicini, R. 2004, A\&A, 426, 809

Sneden, C. A. 1973, Ph.D. Thesis

Soderblom, D. R. 1983, ApJS, 53, 1

Soderblom, D. R., Fedele, S. B., Jones, B. F., Stauffer, J. R., \& Prosser, C. F. 1993, AJ, 106, 1080

Sousa, S. G., Santos, N. C., Mayor, M., et al. 2008, A\&A, 487, 373

Sousa, S. G., Fernandes, J., Israelian, G., \& Santos, N. C. 2010, A\&A, 512, L5

Takeda, Y., \& Tajitsu, A. 2009, PASJ, 61, 471

Takeda, Y., Kawanomoto, S., Honda, S., Ando, H., \& Sakurai, T. 2007, A\&A, 468,663

Takeda, Y., Honda, S., Kawanomoto, S., Ando, H., \& Sakurai, T. 2010, A\&A, 515, A93

Valenti, J. A., \& Fischer, D. A. 2005, ApJS, 159, 141

VandenBerg, D. A., Gustafsson, B., Edvardsson, B., Eriksson, K., \& Ferguson, J. 2007, ApJ, 666, L105

Vardavas, I. M. 2005, MNRAS, 363, L51

Wright, J. T., Marcy, G. W., Butler, R. P., \& Vogt, S. S. 2004, ApJS, 152, 261

Xiong, D. R., \& Deng, L. 2009, MNRAS, 395, 2013

Yadav, R. K. S., Bedin, L. R., Piotto, G., et al. 2008, A\&A, 484, 609

Yi, S., Demarque, P., Kim, Y., et al. 2001, ApJS, 136, 417 